\title{
Interplay effects during enhanced dynamic wedge deliveries
}

\author{
M.B. Kakakhel ${ }^{a, c}$, T. Kairn ${ }^{a, b}$, J. Kenny ${ }^{b}$, K. Seet ${ }^{d}$, \\ A.L. Fielding ${ }^{a}$, J.V. Trapp ${ }^{a, *}$
}

\begin{abstract}
a School of Chemistry, Physics \& Mechanical Engineering, Queensland University of Technology, GPO Box 2434, Brisbane, Qld 4001, Australia

b Premion, The Wesley Medical Centre, Suite 1, 40 Chasely St, Auchenflower, Qld 4066, Australia

'Department of Physics and Applied Mathematics, DPAM, Pakistan Institute of Engineering and Applied Sciences, PO Nilore, Islamabad 45650, Pakistan

${ }^{\mathrm{d}}$ Radiation Oncology, Princess Alexandra Hospital, 199 Ipswich Road, Woolloongabba, Brisbane, Qld 4102, Australia
\end{abstract}

Received 9 January 2012; received in revised form 1 March 2012; accepted 24 April 2012 Available online 26 May 2012

\section{KEYWORDS}

Interplay effects;

Dynamic wedges;

Tumor motion;

Fractionated delivery

\begin{abstract}
In this study the interplay effects for Enhanced Dynamic Wedge (EDW) treatments are experimentally investigated. Single and multiple field EDW plans for different wedge angles were delivered to a phantom and detector on a moving platform, with various periods, amplitudes for parallel and perpendicular motions. A four field 4D CT planned lung EDW treatment was delivered to a dummy tumor over four fractions. For the single field parallel case the amplitude and the period of motion both affect the interplay resulting in the appearance of a step function and penumbral cut off with the discrepancy worst where collimator-tumor speed is similar. For perpendicular motion the amplitude of tumor motion is the only dominant factor. For large wedge angle the dose discrepancy is more pronounced compared to the small wedge angle for the same field size and amplitude-period values. For a small field size i.e. $5 \times 5 \mathrm{~cm}^{2}$ the loss of wedged distribution was observed for both $60^{\circ}$ and $15^{\circ}$ wedge angles for parallel and perpendicular motions. Film results from 4D CT planned delivery displayed a mix of over and under dosages over 4 fractions, with the gamma pass rate of $40 \%$ for the averaged film image at 3\%/1 mm DTA (Distance to Agreement). Amplitude and period of the tumor motion both affect the interplay for single and multi-field EDW treatments and for a limited (4 or 5) fraction delivery there is a possibility of non-averaging of the EDW interplay. (c) 2012 Associazione Italiana di Fisica Medica. Published by Elsevier Ltd. All rights reserved.
\end{abstract}

\footnotetext{
* Corresponding author. Tel.: +61 (0) 731381386; fax: +61 (0) 731389079.

E-mail address: j.trapp@qut.edu.au (J.V. Trapp).
} 


\section{Introduction}

Tumor motion in radiation therapy can be classified as inter-fractional (between treatment fractions) and intrafractional (during the treatment delivery). Intra-fractional organ motion can result in two types of effects, the dose blurring effect, caused by the non uniform dose distribution delivered to a moving tumor resulting in the over/ under dosage [1,2], and the interplay effect, observed during dynamic delivery with intensity modulated radiation therapy (IMRT) $[3,4]$ or enhanced dynamic wedges (EDWs) [5]. The interplay effect results from similar velocities of tumor motion and collimators causing a regular perturbation in the intended dose distribution.

Respiratory intra-fractional organ motion has been studied [6-9] and for lung tumors up to $5 \mathrm{~cm}$ of motion has been observed [10]. Pemler et al. [5] using numerical methods studied the impact of wedge angle, amplitude of organ motion, beam energy, dose rate and the phase of respiratory cycle on the interplay effects using single field treatments with an EDW moving in the cranio-caudal direction only. In this study a single fraction delivery was studied using treatment planning software. The dose discrepancies were highest when collimator and organ motions were comparable in magnitude. Sidhu et al. [11] also investigated the interplay effects for a two field breast delivery over a single fraction; comparing physical wedges, dynamic wedges and IMRT plans for three collimator speeds where tumor motion was in the anterior posterior direction. They also concluded that the deviations from the static case are highest when the collimator and tumor motion are similar in magnitude.

For IMRT treatments the interplay effects have been reported to start averaging out within the first five fractions of the treatment [12-14]. IMRT and VMAT have replaced 3D conformal radiotherapy treatments (3D CRT) for several anatomical sites $[14,15]$. However despite their potential advantage it is impractical to treat every patient using these modalities, as there are associated issues such as cost, proper dose verification and QA etc. which amounts to a lot of work for the physicist in the clinic. Therefore 3D CRT treatments employing dynamic wedges is still a standard in radiotherapy centres [16]. Despite detailed interplay studies for various IMRT techniques, there is a lack of experimental data for multiple field fractionated EDW deliveries. It is important to examine EDW-based interplay effects since a large number of non IMRT treatments employ dynamic wedges. For this study we experimentally examine the effects of interplay between EDW motion and patient motion, for single and multiple field treatments.

\section{Methods and materials}

\section{Equipment}

Dynamic wedges employ the movement of beam shaping collimators with the help of computer control to generate a wedged dose profile [17]. Varian (Varian Oncology Systems, Palo Alto, CA) has upgraded its dynamic wedge implementation to EDWs whereby several improvements have been introduced [18]. These include the ability to generate symmetric and asymmetric wedged fields, availability of more wedge angles, an increased field size up to $30 \mathrm{~cm}$, and fewer segmented treatment tables per photon energy (STT) etc. The wedge was commissioned according to the vendor's EDW Implementation Guide (Varian Medical Systems, Palo Alto, USA) [18]. Commissioning measurements included verification of jaw motions, field size definitions and wedged field shapes using both the light field and the radiation field. Additionally, dose measurements required for treatment planning were obtained using a linear array of ion chambers immersed in water. These dose measurements included profiles for all wedge angles at various depths, as well as depth-dose measurements and wedge factor evaluations.

The present study was conducted in three parts. Throughout the course of investigation the tumor motion was simulated with a respiratory gating motion platform (Standard Imaging, Middleton, USA), with a single (1D) motion axis. This platform can simulate longitudinal movement between $5 \mathrm{~mm}$ and $40 \mathrm{~mm}$ in $5 \mathrm{~mm}$ increments. The frequency of motion can be adjusted from 2 to $6 \mathrm{~s}$ with increments of $0.5 \mathrm{~s}$. The MapCHECK 2 (Sun Nuclear Corp., Melbourne, FL, USA) diode array and EBT2 GAFchromic films (International Specialty Products, Wayne, USA) were used to obtain 2D dose images and lateral dose profiles. The MapCHECK 2 is a two-dimensional diode array consisting of 1527 SunPoint diode detectors arranged in $32 \times 26 \mathrm{~cm}$ matrix. The detector spacing is $7.7 \mathrm{~mm}$ with an active detector area of $0.64 \mathrm{~mm}^{2}$. The device has a buildup and backscatter to the active detectors of $2.0 \pm 0.1$ and $2.75 \pm 0.1 \mathrm{~g} / \mathrm{cm}^{3}$ respectively.

The EBT2 films were scanned using an Epson Perfection V700 Photo flatbed scanner (Seiko Epson Corp., Nagano, Japan) operating in transmission mode according to a previously reported protocol $[19,20]$. Each sheet of film was scanned before and after irradiation, elevated above the glass surface of the scanner, and analysed in the red-channel, without applying a blue-channel correction. A set of small $\left(5 \times 5 \mathrm{~cm}^{2}\right)$ pieces of film were irradiated to known doses between 0 and $450 \mathrm{cG} \mathrm{y}$, and the resulting optical density data were plotted against delivered dose and used to generate a sensitometric curve for the film, which was fitted using a cubic polynomial. The net optical densities determined in each film image obtained during the interplay study were then converted into measurements of dose using the sensitometric curve, producing two-dimensional dose maps of each radiation treatment examined.

For second and third parts of the study, EBT2 films were placed in an IMRT dose verification phantom (Standard Imaging, Middleton, USA) with lung inserts replaced with custom made inserts and a place holder for a dummy tumor. It also incorporates inhomogeneity structures with bone and lung equivalents. Radiation was delivered using a Varian iX linear accelerator, producing a $6 \mathrm{MV}$ photon beam.

Results were compared qualitatively using visual profile comparisons and quantitatively using $2 \mathrm{D}$ gamma analysis [21,22] with $3 \% / 3 \mathrm{~mm}$ and $3 \% / 1 \mathrm{~mm}$ DTA (Distance to agreement) criteria. For image manipulation and registration, Matlab (version 7.8.0.342, The MathWorks, Natick, MA, USA) and ImageJ (Version 1.45d, Rasband, W.S., ImageJ, U. S. National Institutes of Health, Bethesda, Maryland, USA) were used. 


\section{Single field deliveries to a $2 \mathrm{D}$ array}

For all measurements 200 monitor units (MU) were delivered at $600 \mathrm{MU} / \mathrm{min}$ with Y1-IN wedge orientation and $0^{\circ}$ gantry angle to the MapCHECK 2 diode array. The effect of the amplitude and the period of phantom motion were investigated for a $45^{\circ}$ wedge delivery as it is one of the commonly used wedge angles. Two geometries were investigated, first where the phantom-Y-jaw motion was parallel and second where phantom-Y-jaw motion was perpendicular. The platform was always moving in the longitudinal couch direction (for perpendicular motion the collimator was rotated).

The phantom was initially irradiated with a $20 \times 20 \mathrm{~cm}^{2}$ field, while stationary, with the MapCHECK 2 placed on the respiratory platform under $10 \mathrm{~cm}$ of water-equivalent plastic (No additional backscatter was used). The platform was set in motion and further profiles were obtained, varying the amplitude $(10-40 \mathrm{~mm}$, in $10 \mathrm{~mm}$ step) and period values $(3.0,4.5$ and $6.0 \mathrm{~s})$. These period values represented either a faster $(3.0,4.5 \mathrm{~s})$ or $\operatorname{similar}(6.0 \mathrm{~s})$ tumor speed relative to the collimator. The collimator speed was close to $10 \mathrm{~mm} / \mathrm{s}$. For each amplitude-period combination a single static and motion profile was acquired. An amplitude-period was identified from the previous set of measurements (hereafter referred to as the worst case motion parameters), where for the $45^{\circ}$ wedge angle the greatest difference between the static phantom and the moving-phantom profiles was observed.

The effects of wedge angle and field size were assessed employing the worst case motion parameters for parallel and perpendicular phantom-collimator motions. Large and small wedge angles (for the purpose of this study large refers to $60^{\circ}$ and small to $\left.15^{\circ}\right)$ and three field sizes $\left(20 \times 20 \mathrm{~cm}^{2}\right.$, $10 \times 10 \mathrm{~cm}^{2}$ and $5 \times 5 \mathrm{~cm}^{2}$ ) were examined. For a particular field size-wedge angle combination a single static and motion profile was acquired for parallel and perpendicular motions. Furthermore a fast moving tumor scenario was examined (2.0 s period, tumor speed was greater compared to the 3.0 and $4.5 \mathrm{~s}$ period values) for a $20 \times 20 \mathrm{~cm}^{2}$ delivery with $40 \mathrm{~mm}$ amplitude employing a $45^{\circ}$ EDW. Finally to assess the impact of averaging in a multi-fractionated case a single field $\left(45^{\circ}\right.$, $20 \times 20 \mathrm{~cm}^{2}$, worst case motion parameters and parallel phantom-collimator motion) profile was delivered over 5 fractions and qualitatively compared with the single fraction motion delivery. For data processing the MapCHECK 2 inbuilt software was used with its default linear interpolation scheme to generate the dose maps from the measurement points. The perpendicular dose maps were plotted using the inbuilt interpolation scheme in the Matlab software.
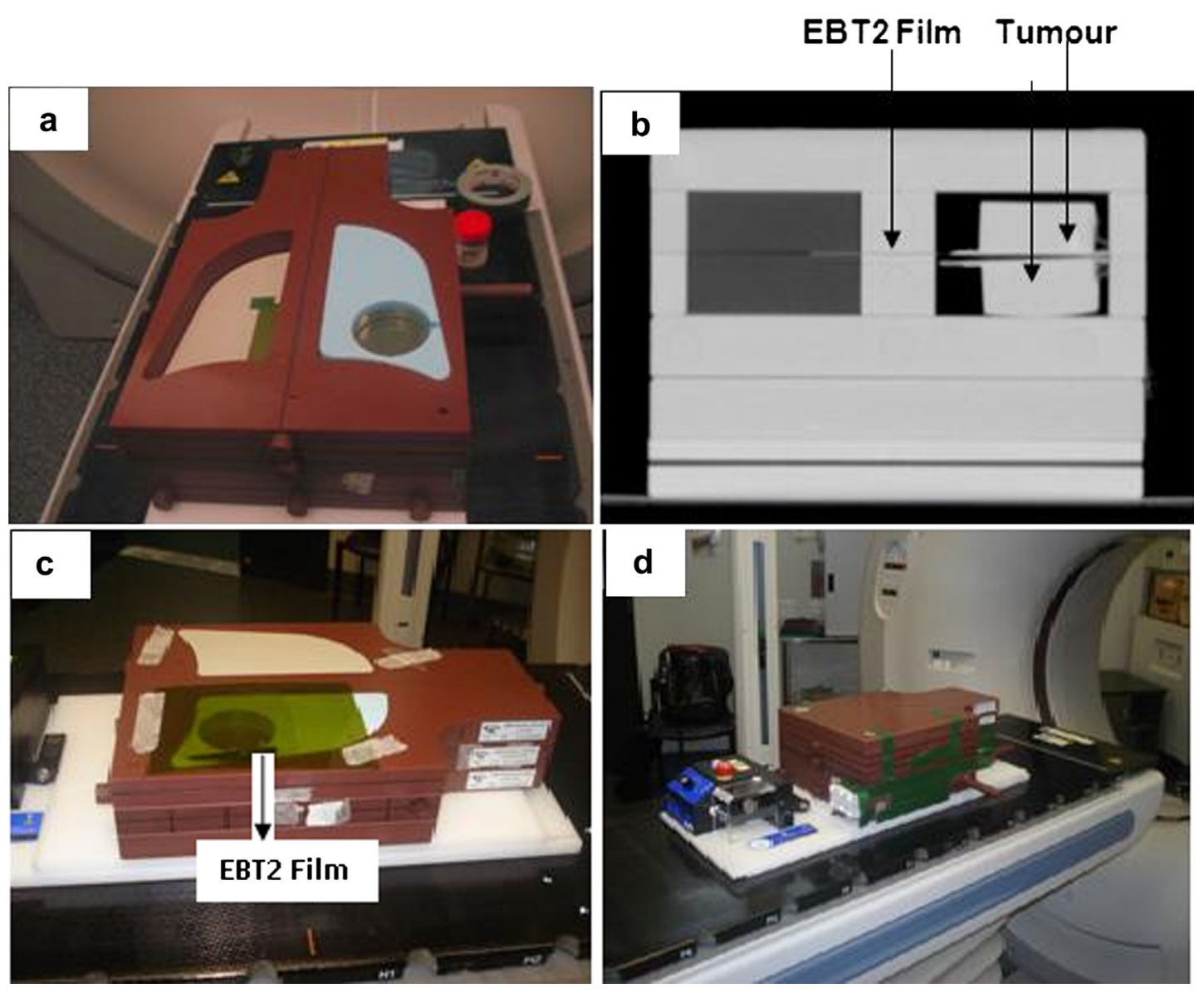

Figure 1 a) Lung insert and tumor in the IMRT phantom b) Tumor film geometry c) EBT2 film placed on top of the tumor in the lung insert d) Overall experimental setup showing the IMRT phantom placed on top of the respiratory platform. Platform motion of direction is along the couch's main axis. 


\section{Patient plans delivered to a 2D diode array and film}

Three existing clinical patient plans (A single breast and two lung plans) were delivered to MapCHECK 2 and the EBT2 film and dose images obtained with the static and moving phantom were compared using gamma analysis. The individual fractions were delivered to the static phantom and to the phantom moving with the worst case motion parameters. For all patient plans the tumor motion was simulated in the superior-inferior direction.

The first plan was a breast case. The MapCHECK2 was placed on the moving platform (without any additional backscatter) for a single fraction, and 2 tangential wedged fields $\left(25^{\circ}\right.$ and $\left.20^{\circ}\right)$ were delivered from their planned gantry angles (treatment parameters presented in Table e1 - supplementary materials). The phantom motion was in
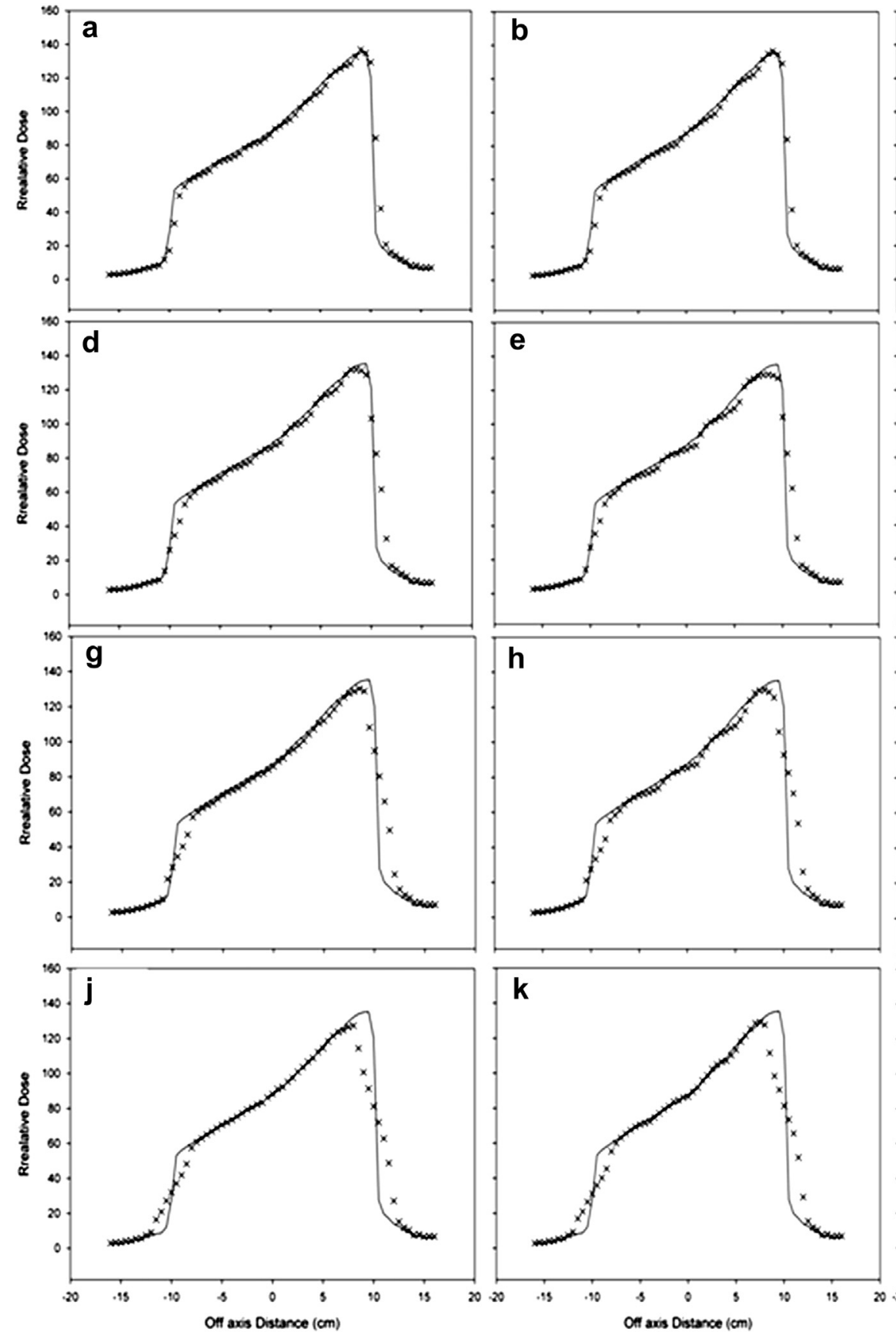
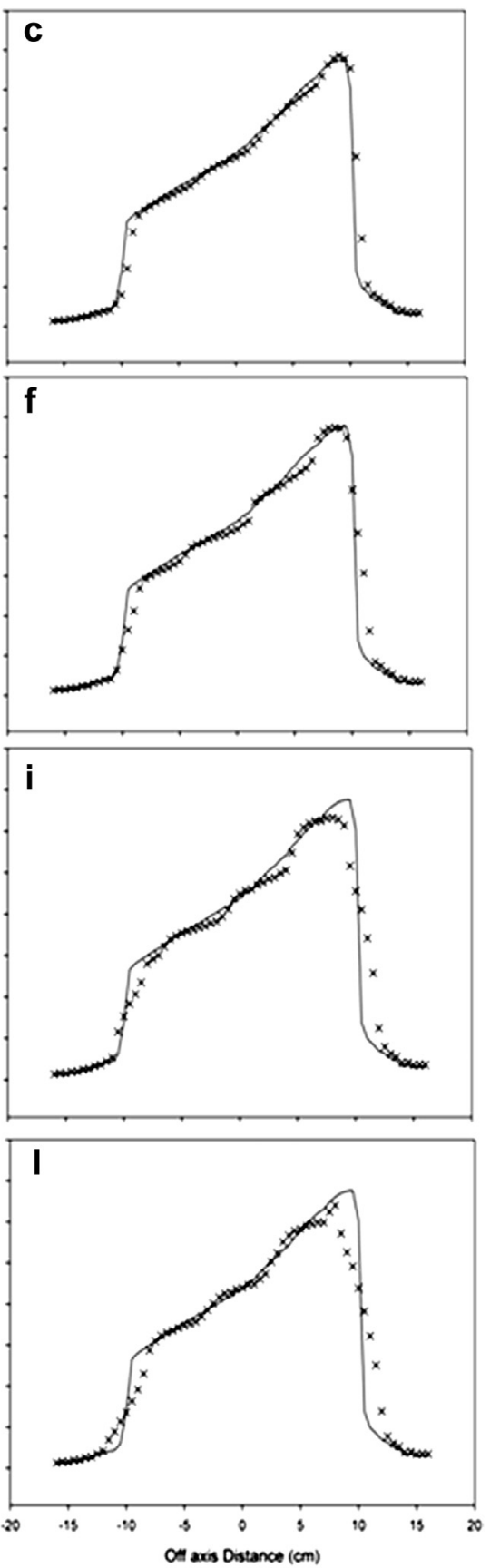

Figure 2 Effect of varying the amplitude and period of motion for a $20 \times 20 \mathrm{~cm}^{2} 45^{\circ}$ EDW with parallel phantom-collimator motion using the MapCHECK 2 diode array where linear interpolation was used to generate dose maps from the diode measurements. Continuous line represents the static and symbols $(\times)$ represent the motion cases respectively (a) $10 \mathrm{~mm}-3.0 \mathrm{~s}(\mathrm{~b})$ $10 \mathrm{~mm}-4.5 \mathrm{~s}$ (c) $10 \mathrm{~mm}-6.0 \mathrm{~s}$ (d) $20 \mathrm{~mm}-3.0 \mathrm{~s}$ (e) $20 \mathrm{~mm}-4.5 \mathrm{~s}$ (f) $20 \mathrm{~mm}-6.0 \mathrm{~s}$ (g) $30 \mathrm{~mm}-3.0 \mathrm{~s}$ (h) $30 \mathrm{~mm}-4.5 \mathrm{~s}$ (i) $30 \mathrm{~mm}-6.0 \mathrm{~s}$ (j) $40 \mathrm{~mm}-3.0 \mathrm{~s}$ (k) $40 \mathrm{~mm}-4.5 \mathrm{~s}$ (l) $40 \mathrm{~mm}-6.0 \mathrm{~s}$. 
the superior-inferior direction while the wedge direction was perpendicular to the phantom motion. The second delivered plan was a four field lung EDW (combination of $45^{\circ}$ and $20^{\circ}$ ) treatment. This plan was delivered to MapCHECK 2 over a single fraction. The plan parameters are listed in Table e1-supplementary materials (Lung \#1).

The third plan was a more complex lung case, consisting of five coplanar wedged fields (combination of $45^{\circ}, 25^{\circ}$ and $20^{\circ}$ ) and an additional wedged field with a $320^{\circ}$ couch rotation. This plan was delivered to the IMRT phantom over two fractions with the EBT2 film placed isocentrically between the two lung slabs in the coronal plane. EBT2 spatial resolution/pixel size was $(0.35 \times 0.35) \mathrm{mm}^{2}$. The IMRT phantom was placed along the couch identical to the setup in Fig. 1 (a) (treatment parameters Table e1 supplementary materials, Lung \#2).

\section{A multi-fractionated 4D CT planned delivery to films}

The lung inserts in the in the IMRT phantom were replaced with custom made foam inserts with a place holder for a dummy tumor (a cylindrical tumour made up of Polyethylene terephthalate (PET) container with a diameter of $7 \mathrm{~cm}$ and height of $2.5 \mathrm{~cm}$-filled with dosimetric gel), as shown in Fig. 1 (a). EBT2 film was placed between the two layers of the tumor in the coronal plane, as shown in Fig. 1 (b) and (c). The overall experimental setup is shown in Fig. 1 (d) where the IMRT phantom is placed on the respiratory platform moving along the main axis of the couch.

The phantom was scanned using a Toshiba Aquilion LB 4D CT scanner using the Varian's real time position monitoring (RPM) system and the 4D CT images were binned into ten respiratory phases. The scan data was imported into the iPlanNET (Ver 2.5, BrainLab AG, Feldkirchen Germany) for sorting and a GTV was outlined based on a maximum intensity projection (MIP) reconstruction of the 10 respiratory phased correlated CT scans. The MIP CT was exported to Eclipse-External Beam Planning 8.6.17 (Varian, Milpitas, USA) for dose calculation using the Anisotropic Analytical Algorithm (AAA), where dose grid size was $2 \mathrm{~mm} \times 2 \mathrm{~mm}$.

A four field EDW $\left(60^{\circ}\right.$ and $\left.30^{\circ}\right)$ plan was generated (Lung patient \# 3, Table e1-supplementary materials) with a prescribed dose of $2.0 \mathrm{~Gy} /$ fraction. This plan was delivered over 4 fractions using worst case motion parameters. The collimator-phantom motion was not phase matched (i.e. organ position at the time when collimator starts moving) because of the negligible phase shift dependence reported earlier [5].

The EBT2 films (with pixel size of $0.35 \mathrm{~mm} \times 0.35 \mathrm{~mm}^{2}$ ) were scanned and the measured dose distributions were averaged to produce a combined image after delivering four fractions. Motion and static images were compared using gamma analysis (3\%/1 mm DTA) and dose-area histogram (DAH).

\section{Results}

\section{Single field deliveries to a 2D diode array}

\section{Effect of amplitude and period for parallel phantom- collimator motion}

In Fig. 2 (a) to (l) a comparison of $20 \times 20 \mathrm{~cm}^{2}$ static and motion profiles along the central axis (instead of the entire dose maps) is presented for a $45^{\circ}$ delivery with multiple amplitudes (10-40 mm) and periods (3.0, 4.5 and $6.0 \mathrm{~s})$. Figure 2 shows that both the amplitude (down the column) and period (across the row) of the phantom motion affect the shape of the profiles.

The static and dynamic profiles show increasing disagreement as the period approaches $6.0 \mathrm{~s}$ where the platform and collimator speeds are nearly similar. Two types of effects can be observed from the profiles in Fig. 2, firstly penumbral blurring, and secondly the appearance of a step like function within the field. The blurring of the penumbra increases with increasing the amplitude of motion (Fig. 2 (a) (d) (g) and (j)) and also causes a drop-off in the peak delivered dose. For any given amplitude, with increasing period the step function becomes more pronounced. Table 1 shows the results of a gamma analysis on the dose maps for $3 \% / 3 \mathrm{~mm}$ and $3 \% / 1 \mathrm{~mm}$ DTA where it can be observed that for any given amplitude the $6.0 \mathrm{~s}$ period results in the lowest pass rate, and that for all periods the gamma pass rate decreases with increasing amplitude.

Amplitude and period of phantom motion are both responsible for dose degradation in case of parallel phantom-collimator motion.

\section{Effect of amplitude and period variation for perpendicular phantom-collimator motion} The effect of phantom motion that is perpendicular to the wedge motion appears as a broadening of the penumbra only, with no major dependence on the period (Fig. e1supplementary materials). Data shown in Table 1 indicates

Table 1 Gamma analysis pass rates with a $3 \% / 3 \mathrm{~mm}$ and $3 \% / 1 \mathrm{~mm}$ acceptance criteria for parallel and perpendicular deliveries.

\begin{tabular}{|c|c|c|c|c|c|c|c|c|c|c|c|c|}
\hline \multirow{3}{*}{$\begin{array}{l}\text { Period (s) } \\
\text { Amplitude } \\
(\mathrm{mm})\end{array}$} & \multicolumn{4}{|l|}{3.0} & \multicolumn{4}{|l|}{4.5} & \multicolumn{4}{|l|}{6.0} \\
\hline & \multicolumn{2}{|c|}{ Parallel } & \multicolumn{2}{|c|}{ Perpendicular } & \multicolumn{2}{|c|}{ Parallel } & \multicolumn{2}{|c|}{ Perpendicular } & \multicolumn{2}{|c|}{ Parallel } & \multicolumn{2}{|c|}{ Perpendicular } \\
\hline & $\begin{array}{l}3 \% / \\
3 \mathrm{~mm}\end{array}$ & $\begin{array}{l}3 \% / \\
1 \mathrm{~mm}\end{array}$ & $\begin{array}{l}3 \% / \\
3 \mathrm{~mm}\end{array}$ & $\begin{array}{l}3 \% / \\
1 \mathrm{~mm}\end{array}$ & $\begin{array}{l}3 \% / \\
3 \mathrm{~mm}\end{array}$ & $\begin{array}{l}3 \% / \\
1 \mathrm{~mm}\end{array}$ & $\begin{array}{l}3 \% / \\
3 \mathrm{~mm}\end{array}$ & $\begin{array}{l}3 \% / \\
1 \mathrm{~mm}\end{array}$ & $\begin{array}{l}3 \% / \\
3 \mathrm{~mm}\end{array}$ & $\begin{array}{l}3 \% / \\
1 \mathrm{~mm}\end{array}$ & $\begin{array}{l}3 \% / \\
3 \mathrm{~mm}\end{array}$ & $\begin{array}{l}3 \% / \\
1 \mathrm{~mm}\end{array}$ \\
\hline 10 & 95 & 92 & 96 & 94 & 94 & 90 & 95 & 84 & 92 & 89 & 95 & 84 \\
\hline 20 & 90 & 87 & 91 & 89 & 87 & 83 & 91 & 89 & 87 & 83 & 91 & 89 \\
\hline 30 & 87 & 85 & 87 & 85 & 81 & 80 & 86 & 85 & 79 & 77 & 87 & 85 \\
\hline 40 & 81 & 80 & 82 & 80 & 81 & 79 & 82 & 81 & 80 & 76 & 82 & 81 \\
\hline
\end{tabular}


that the gamma pass rates for perpendicular motion are almost identical at any amplitude irrespective of the period value. By contrast, for any selected period, the gamma pass rates decrease with the increasing amplitude. Evidently, the effects of perpendicular phantom-collimator motion depend on the amplitude rather than the period of phantom motion.
Effect of wedge angle and field size for parallel phantomcollimator motion

In Fig. $3,60^{\circ}$ and $15^{\circ}$ deliveries are compared for three field sizes $\left(20 \times 20 \mathrm{~cm}^{2}, 10 \times 10 \mathrm{~cm}^{2}\right.$ and $5 \times 5 \mathrm{~cm}^{2}$, employing worst case motion parameters). The result of changing the wedge angle from $60^{\circ}$ to $15^{\circ}$, keeping the same amplitudeperiod parameters (Fig. 3 (a) versus (b) and (c) versus (d))
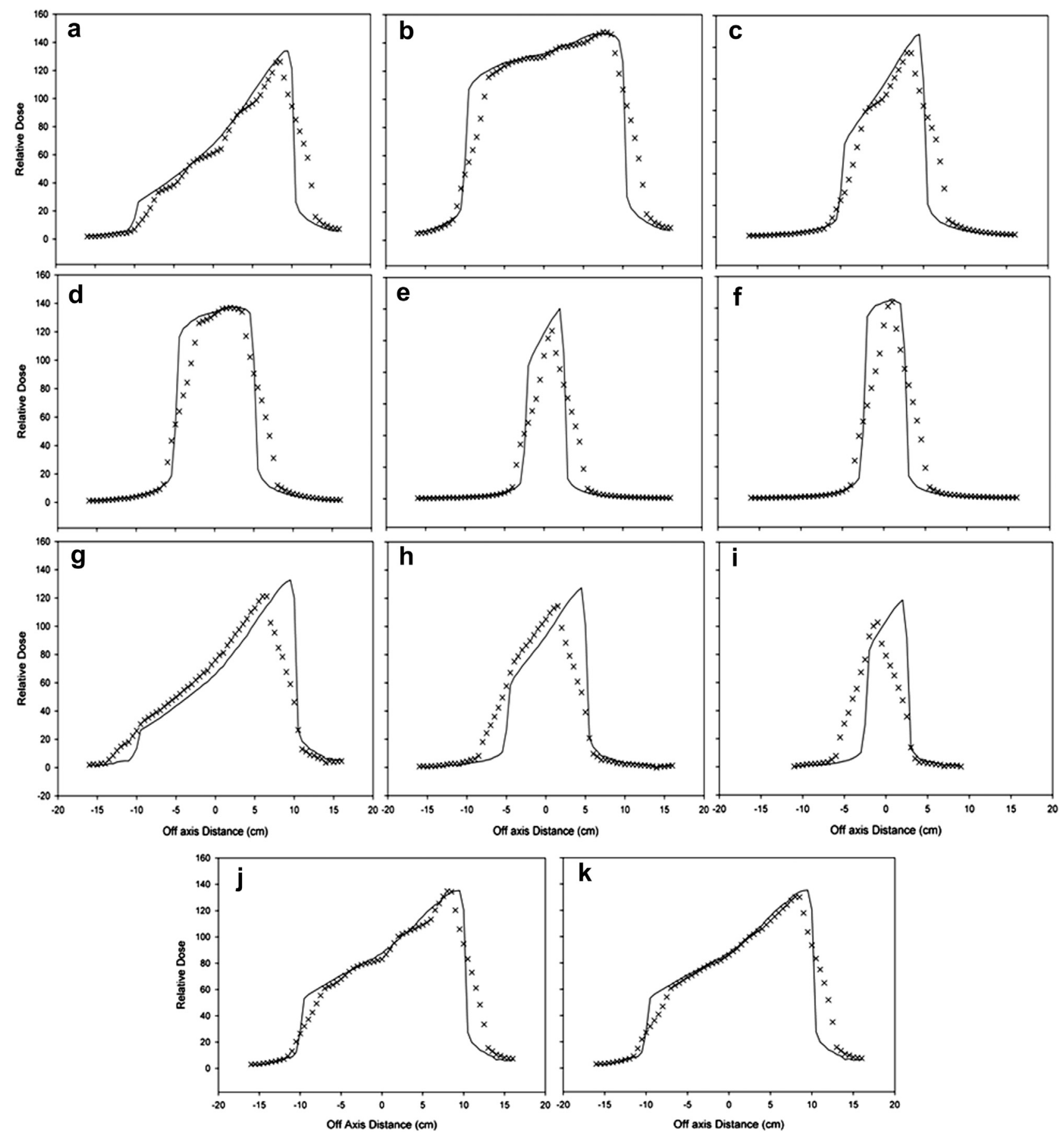

Figure 3 Field size and wedge angle comparison ( $a-f)$, with motion parameters of $40 \mathrm{~mm}$ and $6.0 \mathrm{~s}$ using the MapCHECK 2 diode array where linear interpolation was used to generate dose maps from the diode measurements. continuous line represents the static case and symbols $(\times)$ represent the motion case, a) $20 \times 20 \mathrm{~cm}^{2}-60^{\circ}$ b) $20 \times 20 \mathrm{~cm}^{2}-15^{\circ}$ c) $\left.10 \times 10 \mathrm{~cm}^{2}-60 \mathrm{~d}\right) 10 \times 10 \mathrm{~cm}^{2}$ $-15^{\circ}$ e) $5 \times 5 \mathrm{~cm}^{2}-60^{\circ}$ f) $5 \times 5 \mathrm{~cm}^{2}-15^{\circ}$. A fast moving tumor simulated in $\mathrm{g}-\mathrm{i}$ with motion parameters of $40 \mathrm{~mm}, 2.0 \mathrm{~s} 60 \mathrm{~g}$ ) $20 \times 20 \mathrm{~cm}^{2}$ h) $10 \times 10 \mathrm{~cm}^{2}$ i) $5 \times 5 \mathrm{~cm}^{2}$. Comparison of a single filed 5 fraction delivery for $20 \times 20 \mathrm{~cm}^{2} 45^{\circ}$ j) Single fraction $\mathrm{k}$ ) After 5 fractions. 
showed an increase in the appearance of the step function in the dose profiles for moving detector. For the smaller $5 \times 5 \mathrm{~cm}^{2}$ field size the wedged distribution has been entirely lost for both wedge angles (Fig. 3 (e-f)). The penumbral blurring is evident for all field sizes and both wedge angles, with a more pronounced step function for $60^{\circ}$ wedge angle (Fig. 3 (a)). In Fig. 3 (g-i) profiles for a higher tumor speed compared to the collimator $(2.0 \mathrm{~s}$ period) are shown depicting penumbra blurring without the appearance of the step function.

The effect on a multiple fraction single field delivery Figure $3(j)$ and $(k)$ show the results of a multi-fraction $45^{\circ}$ parallel delivery $\left(20 \times 20 \mathrm{~cm}^{2}\right.$ field using worst case motion parameters). It can be seen that after 5 fractions the step function has smoothed out, however the loss of penumbra remains.

\section{The effect of wedge angle and field size for} perpendicular phantom-collimator motion

For two wedge angles $\left(60^{\circ}\right.$ and $\left.15^{\circ}\right)$ and three field sizes $\left(20 \times 20 \mathrm{~cm}^{2}, 10 \times 10 \mathrm{~cm}^{2}\right.$ and $5 \times 5 \mathrm{~cm}^{2}$, using worst case motion parameters) the perpendicular motion has been compared (Fig. e2 in the supplementary materials). A penumbral broadening can be observed for $20 \times 20 \mathrm{~cm}^{2}$, $10 \times 10 \mathrm{~cm}^{2}$ field sizes for both wedge angles. Also for small field size $\left(5 \times 5 \mathrm{~cm}^{2}\right)$ the wedged distribution is completely lost for $60^{\circ}$ and $15^{\circ}$ wedge angle.

\section{Patient plans delivered to a 2D diode array and film}

The results of the first lung plan delivered over a single fraction are shown in Fig. $4(\mathrm{a}-\mathrm{b})$. Only $79 \%$ of pixels passed a gamma criterion of $3 \%-3 \mathrm{~mm}$ with Fig. 4 (b) demonstrating dose blurring and a change in the shape of the dose distribution.
For the breast plan (Fig. 4 (c) and (d)) motion has resulted in under dosage (compared to the static image) with $89 \%$ pass rate at $3 \% / 3 \mathrm{~mm}$ DTA criterion. The result for the second lung patient are shown in Fig. $4(\mathrm{e}-\mathrm{h})$ where compared to the static case (Fig. 4 (e)) for fraction 1 and 2 (Fig. 4 (f) and (g)) and the average of the two fractions (Fig. $4(\mathrm{~h})$ ), there is visible dose blurring and under dosage also indicated by the $52 \%$ pass rate at $3 \% / 1 \mathrm{~mm}$ DTA.

\section{A multifraction 4DCT planned delivery to films}

The result for the final set of measurements is shown in Fig. 5 where the film dose maps have been generated using Matlab software. For the static image Fig. 5 (a) there is no visible dose degradation. The subsequent 4 motion fractions (Fig. $5(\mathrm{~b}-\mathrm{e})$ ) display a substantial but unequal increase in the irradiated area. In Fig. $5(\mathrm{f}-\mathrm{h})$ the motion averaged images have been presented. The overall result of delivering 4 fractions (Fig. $5(\mathrm{~h})$ ) is a dose image with reduced sharpness of the edges and increased blurring, suggesting an increased dose outside the planned target area.

The gamma analysis at 3\%/1 mm (Fig. $5(j))$ also suggests that in this case, dose averaging over multiple factions has not improved the outcome; the overall pass rate $(40 \%)$ has not improved after 4 fractions. The cumulative dose-area histogram in Fig. $5(\mathrm{k})$ describes the extent of loss of PTV coverage as a result of motion. Figure $5(\mathrm{k})$ indicates that whereas for the static phantom, more than $90 \%$ of the area of the PTV was covered by a dose of $2 \mathrm{~Gy}$, for the moving phantom, less than $70 \%$ of the area of the PTV was covered by a dose of $2 \mathrm{~Gy}$. The TPS image Fig. 5 (i) and static image Fig. 5 (a) look slightly different and this is also confirmed by a pass rate of $80 \%$ at $3 \% / 3 \mathrm{~mm}$. However this difference is because the TPS image has been planned using MIPS.
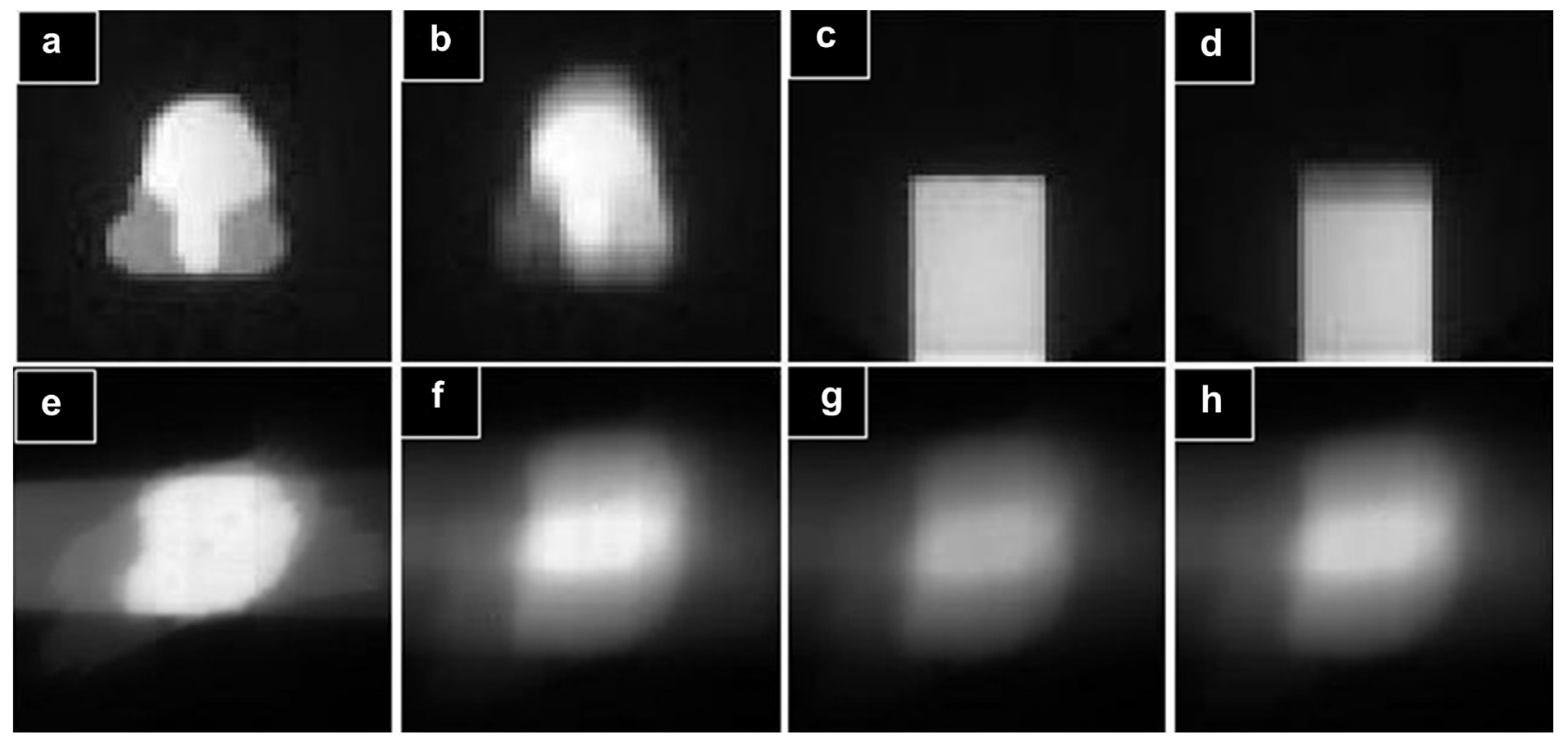

Figure 4 Two lung and a breast plans delivered to MapCHECK 2 diode array and EBT2 films (pixel size of $0.35 \mathrm{~mm} \times 0.35 \mathrm{~mm}{ }^{2}$ ). Motion parameters were $40 \mathrm{~mm}$ and $6.0 \mathrm{~s}$, a) Lung patient \#1-static b) Lung patient \#1-motion c) Breast-static d) Breast-motion. Linear interpolation was used to generate dose maps from the diode measurements. e) Lung patient \#2-static f) Lung patient \#2-motion, fraction $1 \mathrm{~g}$ ) Lung patient \#2-motion, fraction $2 \mathrm{~h}$ ) Lung patient \#2-motion, averaged over two fractions. 

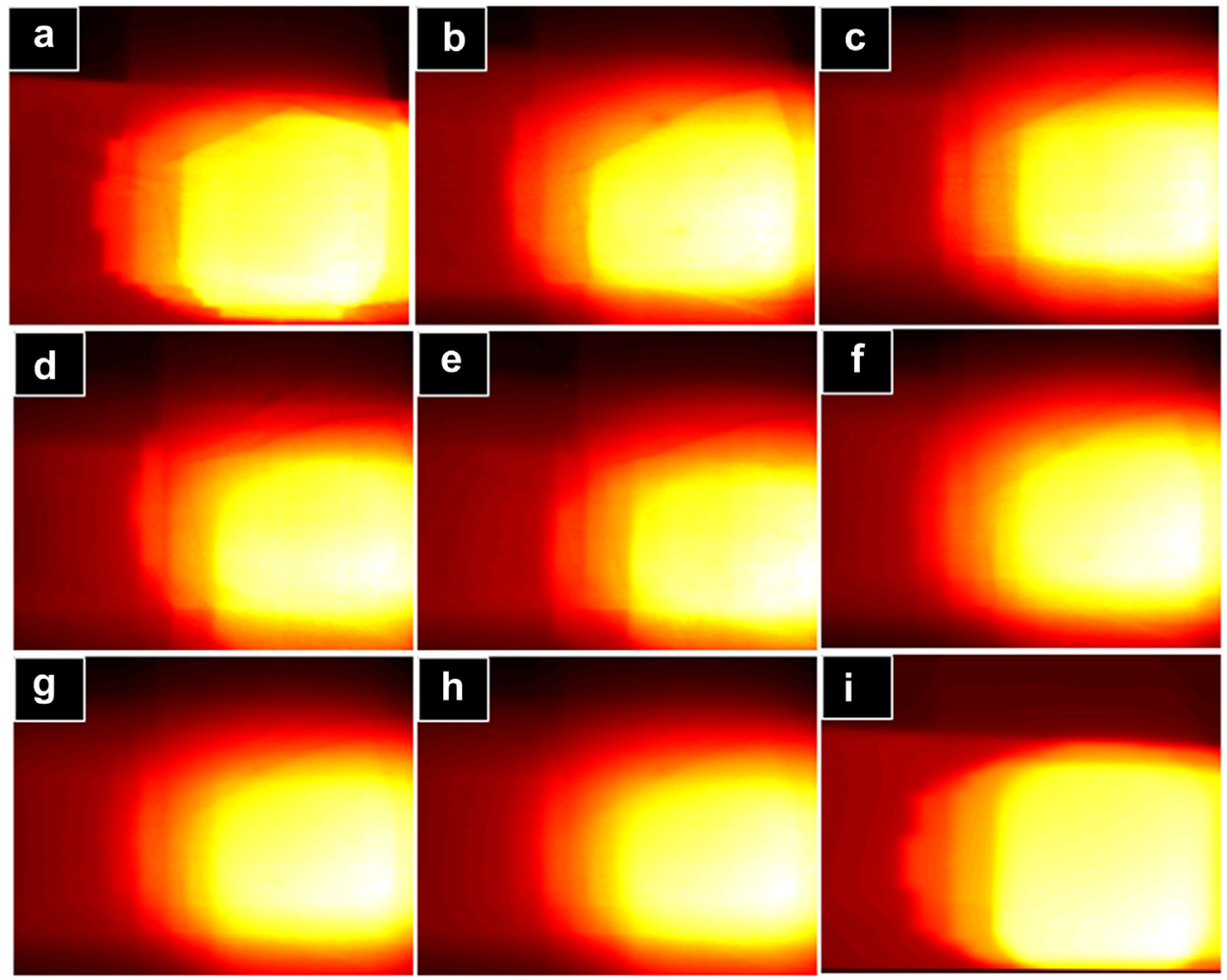

j
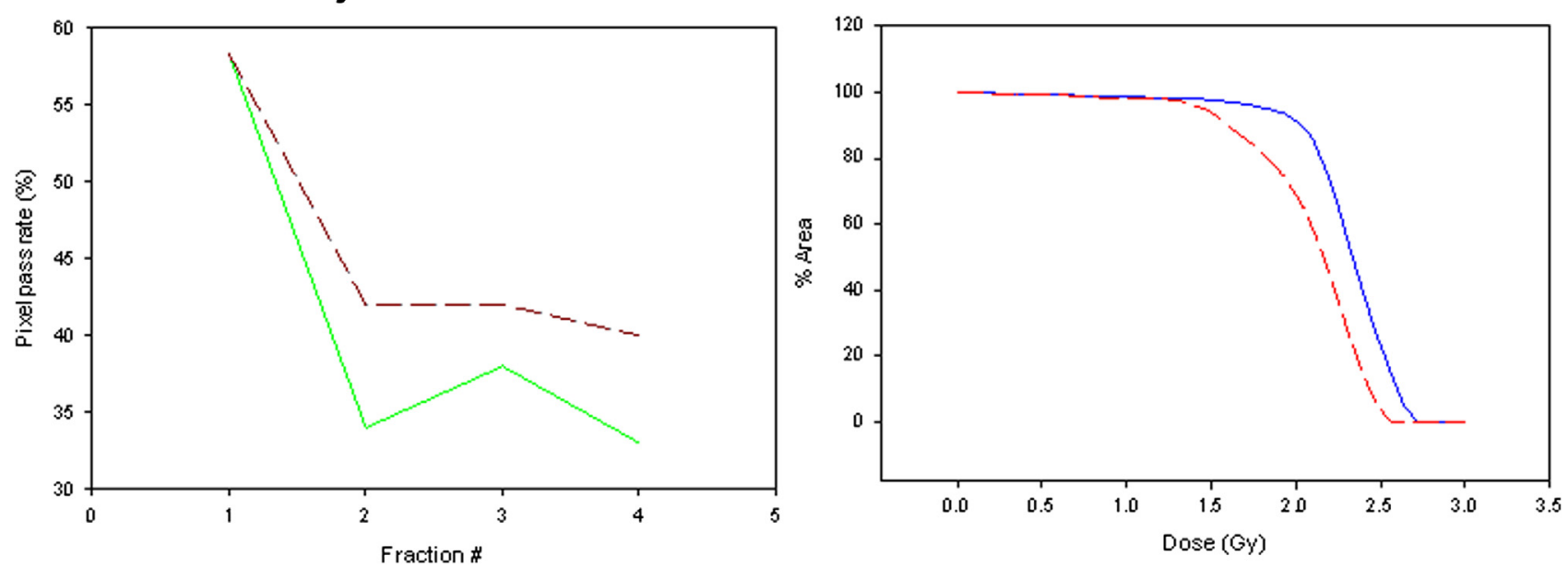

Figure 5 Planned lung patient (Matbalb generated dose maps where dose in Gy is indicated by the color pallete) delivered to the EBT2 film in the IMRT phantom, a) Static b) Motion-fraction $1 \mathrm{c}$ ) Motion-fraction 2 d) Motion-fraction 3 e) Motion-fraction $4 \mathrm{f}$ ) Motion-averaged over fractions $1 \& 2 \mathrm{~g})$ Motion-averaged over fractions 1,2 \& $3 \mathrm{~h}$ ) Motion-averaged Overall 4 fractions i) TPS dose image, j) Gamma analysis, comparing the individual fractions with static case (solid line), comparison of averaged fractions with the static case (dashed line), k) Dose area histogram calculated from the film data for the static (solid blue line) and moving phantom (dashed red line) using the PTV contour from the 4D CT data set.(For interpretation of the references to colour in this figure legend, the reader is referred to the web version of this article.)

\section{Discussion and conclusion}

This study has experimentally investigated the interplay effects for single and multi-field (fractionated) EDW treatments for both parallel and perpendicular motions. For single field parallel delivery, the dose distribution is dependent both on the amplitude and the period of tumor motion, as shown in Fig. 2 with the appearance of a step function along with penumbral cut off. The disagreement between motion and static profiles increases when the collimator-tumor speeds are comparable (6 s case). This observation is consistent with earlier findings by Pemler and Sidhu et al. $[5,11]$. The appearance of a prominent step function as the period approaches the $6 \mathrm{~s}$ value is similar to an interplay-induced step function illustrated by Pemler et al. [5]. 
For perpendicular motion the penumbral cut off increases as the tumor motion amplitude is increased, however the period of tumor motion seems to have little effect as indicated by the constant gamma values across all rows in Table 1 without the appearance of any step function for both $3 \% / 3 \mathrm{~mm}$ and 3\%/1 mm DTA criteria. However it should be noted that further, just because a pass rate is low, it does not necessarily imply an under dosage. The blurringeffect due to motion may result in an under dosage to the target inside the field and an over-dosage outside the field.

Dose differences observed for parallel motion are greater with the appearance of a more prominent step function for a larger wedge angle $\left(60^{\circ}\right)$ (Fig. 3 (a)) due to the steeper dose gradient involved (Fig. 3 (b)). With a reduced field size $\left(5 \times 5 \mathrm{~cm}^{2}\right)$ the wedged dose distribution is lost for both large and small angles $\left(60^{\circ}\right.$ and $\left.15^{\circ}\right)$; this is probably caused by the large amplitude of the tumor motion relative to the sizes of the smaller fields. For perpendicular motion for the same field size, there is a more pronounced penumbral broadening for large wedge angle $\left(60^{\circ}\right)$ compared to the smaller wedge angle $\left(15^{\circ}\right)$. Similar to the parallel case for small field size the wedged distribution is completely lost for both wedge angles.

For the multiple fraction treatment planned specifically for the moving phantom, difference between dose maps (shown in Fig. 5) can be attributed to the random starting phase of the motion. It is clear from Fig. $5(\mathrm{j})$ that for the combined fractions the gamma pass rate (corresponding to images in Fig. 5 (f-h) dashed lines) shows no visible improvement indicating the absence of averaging for this particular study.

With a large number of fractions the interplay may average out, as recently reported for a 25 fraction IMRT delivery by Mohn et al. [23], however as suggested by the authors this averaging will be patient and plan specific. The amplitude value used in this study (i.e. $4 \mathrm{~cm}$ ) has been reported for cases of deep inspiration [24].

There is scope for further study beyond this current proof-of-principle work. The tumor motion here is assumed to be a perfectly sinusoidal 1D motion; however actual lung tumor motion can be irregular with hysteresis [25] and further studies using patient respiratory traces would be beneficial. Similarly the effect of target deformation [26] has not been considered, assuming the tumor motion to be rigid. The interplay can be affected by other factors such as the schematics of the 4D CT, Plan complexity, setup errors etc; however these factors have not been explicitly studied here and were assumed to be the same for both the static and the motion scenarios.

Furthermore interplay can be affected by the application of motion management techniques. These include the use of breath hold techniques in gated deliveries such as deep inspiration breath hold [27], active breathing control [28], self-held breath without respiratory monitoring [29] to name a few. Finally, a simplistic tumor shape has been used in this study and investigation of more complex tumor shapes as reported by Court et al. [13] can be a viable alternative.

\section{Acknowledgments}

The help of John Barrett (QUT Radiological Lab), Simon Belton (QUT BEE workshop) in preparation of the tumor containers/customized lung inserts and Daniel Papworth (Premion) for 4D CT contouring is acknowledged. This work was partly funded by NHMRC (gs1) Project Grant 553012.

\section{Appendix A. Supplementary material}

Supplementary data associated with this article can be found, in the online version, at doi:10.1016/j.ejmp.2012. 04.007 .

\section{Conflict of interest}

There is no reported conflict of interest.

\section{References}

[1] Jiang SB, Pope C, Al Jarrah KM, Kung JH, Bortfeld T, Chen GTY. An experimental investigation on intra-fractional organ motion effects in lung IMRT treatments. Phys Med Biol 2003; 48:1773-84.

[2] Yu CX, Jaffray DA, Wong JW. The effects of intra-fraction organ motion on the delivery of dynamic intensity modulation. Phys Med Biol 1998;43:91-104.

[3] Bortfeld T, Jokivarsi K, Goitein M, Kung JH, Jiang SB. Effects of intra-fraction motion on IMRT dose delivery: statistical analysis and simulation. Phys Med Biol 2002;47:2203-20.

[4] Seco J, Sharp GC, Turcotte J, Gierga D, Bortfeld T, Paganetti $\mathrm{H}$. Effects of organ motion on IMRT treatments with segments of few monitor units. Med Phys 2007;34:923-34.

[5] Pemler P, Besserer J, Lombriser N, Pescia R, Schneider U. Influence of respiration-induced organ motion on dose distributions in treatments using enhanced dynamic wedges. Med Phys 2001;28:2234-40.

[6] Gikas SM, Ellen Y. Deep inspiration breath hold and respiratory gating strategies for reducing organ motion in radiation treatment. Semin Radiat Oncol 2004;14:65-75.

[7] Hiroki S, Yvette S, Kei K, Rikiya O, Shinichi S. Intrafractional tumor motion: lung and liver. Semin Radiat Oncol 2004;14:10-8.

[8] Keall P. 4-dimensional computed tomography imaging and treatment planning. Semin Radiat Oncol 2004;14:81-90.

[9] Keall P, Gig SM, James MB, Richard SE, Kenneth MF, Steve BJ, et al. The management of respiratory motion in radiation oncology report of AAPM task group 76. Med Phys 2006;33: 3874-900.

[10] Chen Q. Fluoroscopic study of tumor motion due to breathing: facilitating precise radiation therapy for lung cancer patients. Med Phys 2001;28:1850-6.

[11] Sidhu S, Sidhu NP, Lapointe C, Gryschuk G. The effects of intrafraction motion on dose homogeneity in a breast phantom with physical wedges, enhanced dynamic wedges, and ssIMRT. Int J Radiat Oncol Biol Phys 2006;66:64-75.

[12] Court LE, Matthew W, Dan I, Ross B, Lee C. Management of the interplay effect when using dynamic MLC sequences to treat moving targets. Med Phys 2008;35:1926-31.

[13] Court LE, Seco J, Lu X-Q, Ebe K, Mayo C, Ionascu D, et al. Use of a realistic breathing lung phantom to evaluate dose delivery errors. Med Phys 2010;37:5850-7.

[14] Bortfeld T. IMRT: a review and preview. Phys Med Biol 2006;51: R363-79.

[15] Otto K. Volumetric modulated arc therapy: IMRT in a single gantry arc. Med Phys 2008;35:310-7.

[16] Khan F. The physics of radiation therapy. 4th ed. Philadelphia: Lippincott Williams \& Wilkins; 2010. 
[17] Leavitt DD, Martin M, Moeller JH, Lee LW. Dynamic wedge field techniques through computer controlled collimator motion and dose delivery. Med Phys 1990;17:87-91.

[18] Varian. Varian oncology systems 2002 C-series clinic enhanced dynamic wedge implementation guide (Palo Alto, CA: Varian); 2002.

[19] Aland T, Kairn T, Kenny J. Evaluation of a Gafchromic EBT2 film dosimetry system for radiotherapy quality assurance. Australas Phys Eng S; 2011:251-60.

[20] Kairn T, Aland T, Kenny J. Local heterogeneities in early batches of EBT2 film: a suggested solution. Phys Med Biol 2010;55:L37-42.

[21] Crowe S, Kairn T, Fielding AL. The development of a Monte Carlo system to verify radiotherapy treatment dose calculations. Radiother Oncol 2009;92 (Supplement 1):S 71-S.

[22] Low DA, Dempsey JF. Evaluation of the gamma dose distribution comparison method. Med Phys 2003;30:2455-64.

[23] Mohn S, Wasbẫ E. Simulation of respiratory motion during IMRT dose delivery. Acta Oncol 2011;50:935-43.

[24] Chen H, Wu A, Brandner ED, Heron DE, Huq MS, Yue NJ, et al. Dosimetric evaluations of the interplay effect in respiratorygated intensity-modulated radiation therapy. Med Phys 2009; 36:893-903.
[25] Seppenwoolde $Y$, Shirato $H$, Kitamura K, Shimizu S, van Herk M, Lebesque JV, et al. Precise and real-time measurement of 3D tumor motion in lung due to breathing and heartbeat, measured during radiotherapy. Int J Radiat Oncol Biol Phys 2002;53:822-34.

[26] Heath E, Seuntjens J. A direct voxel tracking method for fourdimensional Monte Carlo dose calculations in deforming anatomy. Med Phys 2006;33:434-45.

[27] Hanley J, Debois MM, Mah D, Mageras GS, Raben A, Rosenzweig K, et al. Deep inspiration breath-hold technique for lung tumors: the potential value of target immobilization and reduced lung density in dose escalation. Int J Radiat Oncol Biol Phys 1999;45:603-11.

[28] Remouchamps VM, Letts N, Vicini FA, Sharpe MB, Kestin LL, Chen PY, et al. Initial clinical experience with moderate deepinspiration breath hold using an active breathing control device in the treatment of patients with left-sided breast cancer using external beam radiation therapy. Int J Radiat Oncol Biol Phys 2003;56:704-15.

[29] Barnes EA, Murray BR, Robinson DM, Underwood LJ, Hanson J, Roa WHY. Dosimetric evaluation of lung tumor immobilization using breath hold at deep inspiration. Int J Radiat Oncol Biol Phys 2001;50:1091-8. 